\title{
Formules pour le calcul du tassement des sols compressibles par la méthode œedométrique
}

\section{O. COMBARIEU \\ Ingénieur ESTP}

HDR université de Caen 591 E, rue de Strasbourg 76320 Caudebec-lès-Elbeuf
Cet article fournit, pour les principales situations géotechniques courantes, des expressions simples d'utilisation, facilitant le calcul du tassement des couches de sols compressibles à partir des résultats cdométriques classiques. Quelques exemples numériques sont traités, qui montrent, tout particulièrement dans le cadre des techniques d'amélioration des sols, l'importance de la surconsolidation et des nappes d'eau avec la nécessité, pour ces dernières, d'en cerner au mieux les variations.

Mots-clés : œdomètre, tassement, gonflement, nappe, sol compressible, amélioration des sols, inclusions rigides.

\section{Expressions for compressible soils settlement by oedometric method}

This article gives for the main usual geotechnical situations simple expressions, which facilitate the settlement calculation of compressible soil layers, from classical oedometer tests. Some numerical examples are given that show importance of water table's variation, especially in case of use of soil improvement technics.

Key words : oedometer test, settlement, swelling, water table, compressive soil, ground improvement, stiff inclusions. 


\section{Introduction}

Cet article n'a pas pour objet de rappeler la pertinence de la méthode codométrique ni la nécessité de l'utiliser pour prévoir le tassement à long terme des sols compressibles sous des charges de grande étendue. C'est la seule qui peut le permettre sérieusement. Malheureusement, en France, beaucoup l'ont perdue de vue, et quelques raisons expliquent ce constat.

Cette méthode demande une technicité et un soin particulier, puisqu'elle oblige à prélever des sols mous, dans les meilleures conditions possibles. La plus ou moins bonne qualité des carottes ainsi obtenues influe sur le résultat des essais réalisés au laboratoire; en particulier, le souvenir de contraintes anciennes, que le massif de sol a déjà supportées, peut être estompé si toutes les précautions ne sont pas remplies le long de la chaîne des opérations successives, depuis le terrain jusqu'à l'essai en laboratoire.

Ce respect des règles de l'art a un coût, considéré comme logique autrefois, mais que le développement d'essais concurrents, très rapides et peu chers, tend à faire considérer comme prohibitif. Ainsi, l'essai pressiométrique possède effectivement ces qualités, mais n'est malheureusement pas adapté au problème posé. De nombreux exemples (Combarieu, 2006; Lavisse et Schmitt, 2004) montrent les très grandes différences entre les deux approches, en faveur de celle utilisant l'œdomètre. On peut d'ailleurs dire qu'un bon géotechnicien, n'ayant pas d'essais œdométriques à sa disposition, n'a pas besoin d'essais pressiométriques et peut, néanmoins, prévoir un ordre de grandeur raisonnable du tassement.

Aussi, devant la grande et trompeuse simplicité de l'expression $s=\alpha q H / E_{M}$ (où $\alpha$ est le coefficient rhéologique, $\mathrm{E}_{\mathrm{M}}$ le module pressiométrique, $q$ la contrainte apportée par la surcharge et $\mathrm{H}$ l'épaisseur de la couche concernée), il est donné dans cet article des expressions analytiques simples, adimensionnelles, facilitant le calcul du tassement cedométrique pour un certain nombre de situations géotechniques et mécaniques fréquemment rencontrées. Ces expressions, qui ont des formes assez remarquables, sont rapidement et aisément calculables avec une simple calculatrice scientifique.

Si certaines peuvent paraître un peu « lourdes », elles s'avèrent en réalité plus faciles d'emploi que la méthode usuelle et simplificatrice qui consiste à découper le massif en tranches successives pour lesquelles la valeur de la contrainte, au milieu de chacune d'elles, est considérée comme uniforme sur toute l'épaisseur de cette dernière. Comme il est montré plus loin, cette procédure introduit des erreurs très variables suivant le découpage réalisé ; les expressions présentées éliminent ces erreurs.

\section{2}

\section{Notations utilisées}

Le sol est caractérisé, quant à sa compressibilité, par $\mathrm{C}_{\mathrm{C}}$ indice de compressibilité, $\mathrm{C}_{5}$ indice de gonflement et $e_{0}$ indice initial des vides en place.

$$
\text { On pose } \mathrm{k}=0,435 \frac{\mathrm{C}_{\mathrm{c}}}{1+\mathrm{e}_{0}} \text { et } \mathrm{k}^{\prime}=0,435 \frac{\mathrm{C}_{\mathrm{s}}}{1+\mathrm{e}_{0}} \text {. car }
$$

on utilise les logarithmes népériens, notés ln.
Les poids volumiques sont $y$ pour le sol saturé ou le sol humide non saturé (poids volumique apparent) ; $\gamma^{\prime}$ est le poids volumique déjaugé et $\gamma_{\ldots}$ le poids volumique de l'eau.

\section{On a $\gamma$ saturé $=\gamma^{\prime}+\gamma_{w}$}

Les épaisseurs sont notées $\mathrm{H}$ ou h (indicés ou non) pour les valeurs discrètes, et la profondeur z lou z' en Cas de changement d'origine de manière générale, l'axe des z étant orienté vers le bas.

Les contraintes appliquées, généralement uniformes sur de grandes surfaces et provoquant les tassements, sunt notées q (indicé si nécessaire) et sont appelées indiftéremment surcharges ou surcontraintes. Elles peuvent être variables avec z, comme ce sera le cas lors de l'utilisation d'inclusions rigides qui provoquent une variation.

Les contraintes ayant existé puis disparu (surconsolidation) sont notées $\sigma^{\prime}$. Elles étaient, lors de leur existence, des surcontraintes; elles peuvent être également variables avec $z$, comme dans le cas de fluctuation de la nappe. Les fluctuations d'une nappe d'eau sur l'épaisseur partielle d'un sol compressible, génèrent, en effet, une surconsolidation particulière.

Les tassements notés $s(z)$ et $s^{\prime}(z)$ représentent ceux de lá couche d'épaisseur $z$, comprise entre 0 et $z$; s est le tassement, dépendant de $C$ et s' celui dépendant de $\mathrm{C}_{5}$. Ce sont des tassements de consolidation primaire, au sens de la théorie de Terzaghi, utilisée ici.

Par ailleurs, $s$ et $s^{\prime}$ pourront être indicés $\left(s_{\gamma^{\prime}} s_{B}^{\prime} \ldots\right)$ en fonction des couches notées $\mathrm{A}, \mathrm{B}$ indiquées sur les différentes figures.

Pour les expressions donnant s ou s', elles sont repérées par un numéro d'urdre d'apparition croissant, éventuellement indicé avec un ( bis » dans la mesure où l'expression est une variante.

Les expressions donnant le tassement sont d'abord calculées pour une première série de cinq contigurations classiques où le sol reçoit une surcharge q uniforme et constante avec la profondeur z.

Une seconde série de configurations correspond à l'utilisation d'inclusions rigides pour le renforcement de sul compressible, où ce dernier, surchargé en tête, se trouve progressivement déchargé en profondeur du fait de la présence des inclusions.

Dans un but de simplification, les valeurs de tassement étant toutes fournies par une formule générale unique, les expressions donnant ces valeurs n'ont pas toutes été écrites; seuls les éléments, comme explicité au paragraphe 3 , permettant les calculs, sont fournis.

Le tassement total à déterminer est souvent la somme de plusieurs termes, dont certains, tels ceux affectant les tassements de recompression, s'avèrent souvent dans la pratique très faibles et donc négligeables. C'est au géotechnicien à juger de l'intérêt ou non de les calculer en fonction des incertitudes affectant les tassements de compression qui sont, eux, prépondérants.

Enfin, dans les situations où la nappe peut fluctuer, les calculs sont menés pour une nappe en position haute, étant entendu qu'il est tenu compte de sa varia tion dans la détermination des tassements. 


\section{Principe de calcul des tassements}

Il va de soi que la qualité des calculs repose sur l'établissement d'un modèle géotechnique représentatif sur une détermination correcte des diagrammes de contraintes.

Les expressions élémentaires bien connues don nant les tassements sont les suivantes.

Au niveau $z$, sur une couche d'épaisseur dz, règne une contrainte effective $\sigma_{0}^{\prime}(z)$ et où a régné une contrainte $\sigma_{c}^{\prime}-\sigma^{\prime}(z)$ (surconsolidation) ; on applique la surcontrainte $q(z)$.

Si $q(z)>\sigma_{c}^{\prime}$ un tassement élémentaire de compres sion ds se manifeste, tel que :

$$
\frac{1}{\mathrm{k}} \frac{\mathrm{ds}}{\mathrm{dz}}=\ln \frac{\mathrm{q}(\mathrm{z})+\sigma_{0}^{\prime}(\mathrm{z})}{\sigma_{\mathrm{c}}^{\prime}+\sigma_{0}^{\prime}(\mathrm{z})}
$$

auquel s'ajoute un tassement élémentaire de recompression $\mathrm{ds}^{\prime}$ tel que :

$$
\frac{1}{\mathrm{k}^{\prime}} \frac{\mathrm{ds}}{\mathrm{dz}}=\ln \frac{\sigma_{\mathrm{c}}^{\prime}+\sigma_{0}^{\prime}(\mathrm{z})}{\sigma_{0}^{\prime}(\mathrm{z})}
$$

Si $q(z)<\sigma^{\prime}$ seul un tassement de recompression se manifeste, tel que :

$$
\frac{1}{k^{\prime}} \frac{d s^{\prime}}{d z}=\ln \frac{q(z)+\sigma_{0}^{\prime}(z)}{\sigma_{0}^{\prime}(z)}
$$

Au sein d'une couche homogène d'épaisseur z donnée, on a le plus souvent $\sigma_{c}^{\prime}(\mathrm{z})=\sigma_{0}^{\prime}+\gamma^{\prime} z$ où $\sigma_{0}^{\prime}$ représente au sommet de la couche, la contrainte effective en place. Les relations 1, 2 et 3 permettent, après intégration sur l'épaisseur de la couche dans laquelle les inégalités reliant $q(z)$ et $\sigma^{\prime}$ sont partout respectées, de calculer les tassements. La surcharge q(z) peut être variable avec $z$, ce qui est le cas dans la technique de renforcement par inclusions rigides. De même, $\sigma_{c}^{\prime}$ peut être variable avec $\mathrm{z}$, c'est le cas, comme déjà indiqué, lorsque la nappe fluctue régulièrement.

Les expressions auxquelles on a abouti résultent des propriétés de la fonction logarithme. On remarque que si A, B, C, D sont des constantes, on peut écrire successivement:

$$
\frac{1}{\mathrm{H}} \int_{0}^{\mathrm{H}} \ln \frac{\mathrm{A}+\mathrm{Bz}}{\mathrm{Bz}} \mathrm{dz}=\ln \frac{(1+\mathrm{u})^{1+\mathrm{u}}}{\mathrm{u}^{\mathrm{u}}}
$$

et $\frac{1}{\mathrm{H}} \int_{0}^{\mathrm{H}} \ln \frac{\mathrm{A}+\mathrm{Bz}}{\mathrm{C}+\mathrm{Dz}} \mathrm{dz}=\frac{1}{\mathrm{H}} \int_{0}^{\mathrm{H}} \ln \left[\frac{\mathrm{A}+\mathrm{Bz}}{\mathrm{Bz}} \cdot \frac{\mathrm{Dz}}{\mathrm{C}+\mathrm{Dz}} \cdot \frac{\mathrm{B}}{\mathrm{D}}\right] \mathrm{dz}$

$$
\begin{gathered}
=\ln \left[\frac{(1+u)^{1+u}}{u^{u}} \cdot \frac{v^{v}}{(1+v)^{1+v}} \cdot \frac{B}{D}\right] \\
=L\left(u, v, \frac{B}{D}\right), \text { avec } u=\frac{A}{B H} \text { et } v=\frac{C}{D H}
\end{gathered}
$$

Ces transformations de l'intégrale $\mathbf{L}$ ne sont possibles que sous certaines conditions portant sur les signes des valeurs des termes entrant sous le signe somme, auxquels doit satisfaire la fonction logarithme; il en sera a priori toujours ainsi pour les applications traitées.

L'intégrale $\mathrm{L}$ présente la forme générale à laquelle les calculs de tassement mènent, dans la mesure où l'on peut exprimer les contraintes en jeu sous forme linéaire, en particulier pour $q(z)$; si manifestement cette condition n'est pas remplie, le géotechnicien a la possibilité de linéariser par segments pour lesquels les expressions sont alors applicables. Pour les diverses situations étudiées, on considère que ces conditions de linéarité sont remplies.

Dans les diverses configurations ci-après, les formules donnant les tassements sont écrites explicitement lorsqu'elles sont simples; seules les valeurs $u, v$ et B/D à appliquer à l'intégrale L sont clonnées dans les autres cas. Certains cas de figures mènent à $u$ et/ou $v=0$, donnant $(1+u)^{1+u / u^{\nu}}=1$ et/ou $(1+v)^{1+v / v^{v}}=1$, ou bien $B / D=1$.

On convient de maintenir l'écriture symbolique de $L$ avec les trois valeurs $u, v$ et $B / D$, figurant entre parenthèses, tant que $\mathrm{B} / \mathrm{D}$ diffère de 1 . Cette convention évite toute ambiguïté, car les lettres $u, v$ et B/D peuvent avoir, dans le texte, des appellations différentes. On trouvera donc les notations $\mathrm{L}(0,0, \mathrm{~B} / \mathrm{D}=\mathrm{In}$ $\mathrm{B} / \mathrm{D}, \mathrm{L}(\mathrm{u}, 0, \mathrm{~B} / \mathrm{D}), \mathrm{L}(\mathrm{u}, \mathrm{v}, \mathrm{B} / \mathrm{D}) \ldots$ Par contre, si $\mathrm{B} / \mathrm{D}$ vaut 1, on supprime son écriture dans $\mathrm{L}$, ceci dans un but de simplification. Ainsi, on rencontrera $\mathrm{L}(\mathrm{u}, \mathrm{v}), \mathrm{L}(\mathrm{u}, 0) \ldots$ si $\mathrm{B} / \mathrm{D}=1$.

4

Couche de sol normalement consolidée, surchargée sous q

Le sol d'épaisseur H est considéré comme saturé dès la surface ; si ce n'est pas le cas, $\gamma^{\prime}$ est remplacé par $\gamma$. Ce cas correspond dans la pratique à celui du sol naturel noyé.

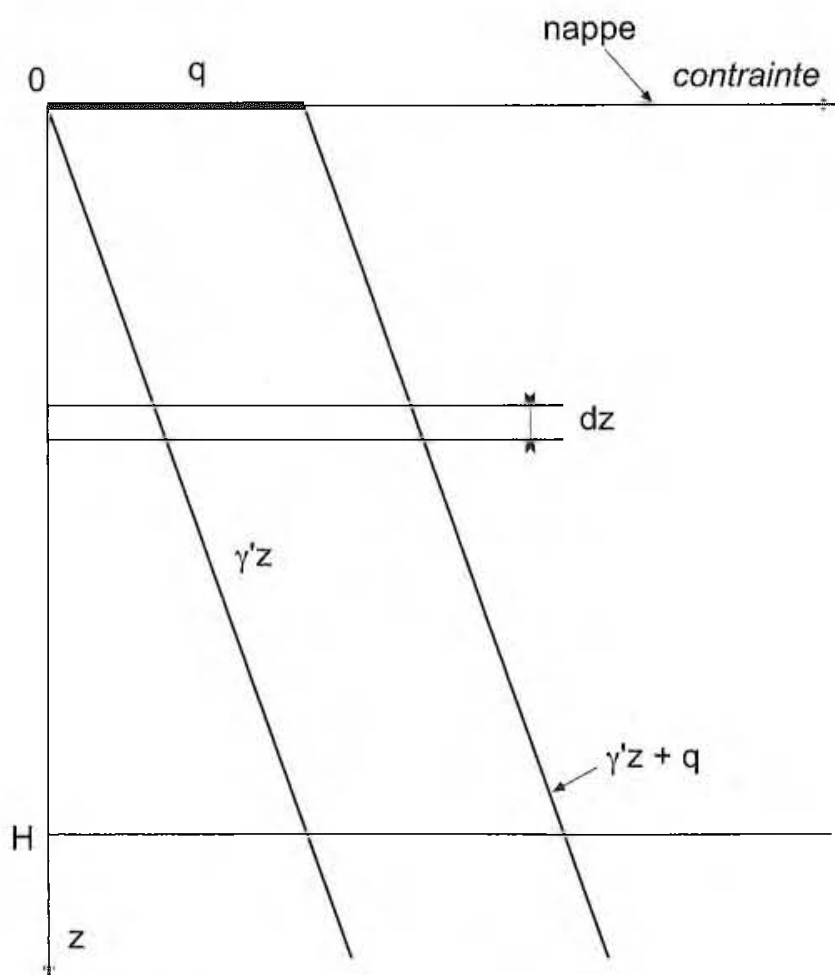

FIG. 1 Sol normalement consolidé, surchargé par q Normally consolidated soil, with surcharge q.

Le tassement ds(z) de la couche élémentaire d'épaisseur dz est donné par :

$$
\frac{d s(z)}{k d z}=\ln \frac{q+\gamma^{\prime} z}{\gamma^{\prime} z}
$$


ce qui peut s'écrire après intégration, en posant $\mathrm{u}=\mathrm{q} / \mathrm{\gamma}^{\prime} \mathrm{H}$ sous la forme simple:

$$
\frac{\mathrm{s}(\mathrm{H})}{\mathrm{kH}}=\ln \frac{(1+\mathrm{u})^{1+\mathrm{u}}}{\mathrm{u}^{\mathrm{u}}}=\mathrm{L}(\mathrm{u}, 0)
$$

On a souvent l'habitude, justifiée lorsque $\mathrm{H}$ est faible, de choisir pour l'intégrale définie entre 0 et $\mathrm{H}$ une valeur moyenne pour le terme $\gamma$ 'z, qui est celle à micouche, soit $\gamma^{\prime} \frac{\mathrm{H}}{2}$; cette pratique fournit une valeur approchée $\mathrm{s}_{a p}(\mathrm{H})=\mathrm{kH} \ln (1+2 \mathrm{u})$, inférieure à $\mathrm{s}(\mathrm{H})$.

On oublie souvent cette condition sur $\mathrm{H}$, qui fait que l'on sous-estime dans des proportions variables le véritable tassement, comme le montrent les valeurs suivantes du rapport $\mathrm{s}(\mathrm{H}) / \mathrm{s}_{\mathrm{ap}}(\mathrm{H})$ en fonction de $\mathrm{u}$ (Tableau I).

\begin{tabular}{|c|c|c|c|c|c|c|c|}
\hline \multicolumn{2}{|c|}{ TABLEAU I } & \multicolumn{6}{|c|}{$\begin{array}{l}\text { Rapport } \mathbf{s} / \mathbf{s}_{\text {apg }} \text { pour différentes valeurs de } \mathbf{u} \text {. } \\
\mathrm{s} / \mathrm{s}_{\mathrm{ap}} \text { ratio for different values for } \mathrm{u}\end{array}$} \\
\hline $\mathrm{u}$ & 5 & 3 & 2 & 1 & 0,5 & 0,25 & 0,17 \\
\hline$s / s_{a p}$ & 1,13 & 1,16 & 1,19 & 1,26 & 1,38 & 1,54 & 1,70 \\
\hline
\end{tabular}

Pour tout ce qui suit, on utilisera la valeur exacte obtenue par intégration.

On peut être amené à calculer le tassement s(z) de la tranche comprise entre 0 et $\mathrm{z}<\mathrm{H}$, ce que l'on peut faire de la même manière en introduisant $u(z)=\frac{q}{\gamma^{\prime} z}$, Mais il peut s'avérer intéressant d'exprimer s(z) en fonction de $\mathrm{H}$, sous la forme :

$$
\frac{\mathrm{s}(\mathrm{z})}{\mathrm{kH}}=\ln \frac{(\mathrm{z} / \mathrm{H}+\mathrm{u})^{\mathrm{z} / \mathrm{H}+\mathrm{u}}}{(\mathrm{z} / \mathrm{H})^{z / H} \cdot \mathrm{u}^{\mathrm{u}}}=\mathbf{L}_{\mathrm{z} / \mathrm{H}}(\mathrm{u}, 0)
$$

où u est défini précédemment (éq. 6). Cette écriture symbolique sera expliquée au paragraphe 5.

\section{5}

\section{Couche de sol normalement consolidée, chargée en tête sous $\sigma^{\prime}$. surchargée sous q}

Ce cas correspond à celui d'un sol de surface d'épaisseur $\mathrm{H}$, déjà chargé, ou d'une couche plus profonde pour laquelle la charge est celle due à la présence du massif sus-jacent.

Le tassement provoqué par $q, s(H)=k \int_{0}^{H} \ln \frac{q+\sigma_{0}^{\prime}+\gamma^{\prime} z}{\sigma_{0}^{\prime}+\gamma^{\prime} z} d z$ S'écrit également :

$$
\frac{\mathrm{s}(\mathrm{H})}{\mathrm{k}}=\int_{0}^{\mathrm{H}} \ln \frac{\mathrm{q}+\sigma_{0}^{\prime}+\gamma^{\prime} \mathrm{z}}{\gamma^{\prime} \mathrm{z}} \mathrm{dz}-\int_{0}^{\mathrm{H}} \ln \frac{\sigma_{0}^{\prime}+\gamma^{\prime} \mathrm{z}}{\gamma^{\prime} \mathrm{z}} \mathrm{dz}
$$

C'est donc la différence entre les tassements tels que définis au paragraphe 4 , d'abord sous une contrainte équivalente à une surcharge $: q+\sigma_{0}^{\prime}$ puis sous la seule contrainte, également assimilable à une surcharge $\sigma_{0}^{\prime}$.

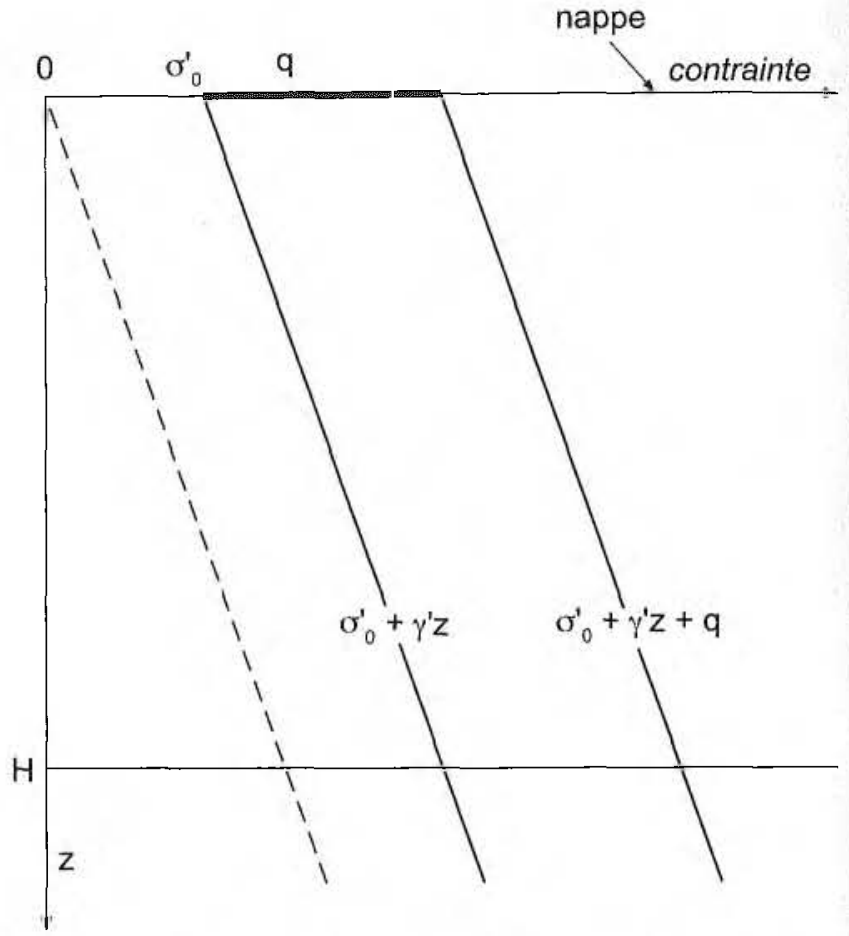

FIG. 2 Sol normalement consolidé, chargé et surchargé par q.

Normally consolidated loaded soil, with surcharge $q$.

Le tassement $\mathrm{s}_{i \mathrm{H})}$ est donc l'accroissement dû à $q$, du tassement qui s'est manifesté autrefois sous $\sigma_{0}^{\prime}$. C'est cet accroissement qui intéresse l'ouvrage à construire.

On peut donc écrire:

$$
\frac{\mathrm{s}(\mathrm{H})}{\mathrm{kH}}=\ln \left[\frac{(1+\mathrm{u})^{1+u}}{\mathrm{u}^{\mathrm{u}}} \cdot \frac{\mathrm{v}^{\mathrm{v}}}{(1+\mathrm{v})^{1+v}}\right]=\mathbf{L}(\mathrm{u}, \mathrm{v})
$$

avec $\mathrm{u}=\left(\mathrm{q}+\sigma_{0,}^{\prime}\right) / \gamma^{\prime} \mathrm{H}$ et $\mathrm{v}=\sigma_{0}^{\prime} / \gamma^{\prime} \mathrm{H}$.

$\mathrm{s}(\mathrm{z})$, tassement de la tranche comprise entre 0 et $\mathrm{z}$, s'exprime directement en fonction de $\mathrm{H}$ par :

$$
\frac{\mathrm{s}(\mathrm{z})}{\mathrm{kH}}=\ln \left[\frac{(\mathrm{z} / \mathrm{H}+\mathrm{u})^{\mathrm{z} / \mathrm{H}+\mathrm{u}}}{\mathrm{u}^{\mathrm{u}}} \cdot \frac{\mathrm{v}^{\mathrm{v}}}{(\mathrm{z} / \mathrm{H}+\mathrm{v})^{\mathrm{z} / \mathrm{H}+\mathrm{v}}}\right]=\mathbf{L}_{\mathrm{z} / \mathrm{H}}(\mathrm{u}, \mathrm{v})
$$

Expression identique à (9), mais dans laquelle on remplace les valeurs 1 par $\mathrm{z} / \mathrm{H}$.

Si $\sigma_{0)}^{\prime}=0$, les relations (9) et (10) redonnent bien (6) et (7) du cas de figure précédent du paragraphe 4.

\section{6}

\section{Couche de sol surconsolidée, chargée en tête et surchargée sous q}

L.e massif a été autrefois surchargé par $\sigma^{\prime}$ contrainte aujourd'hui disparue. Deux expressions différentes s'établissent suivant que q est supérieur ou inférieur à $\sigma^{\prime}$.

Si $q>\sigma_{c}$, le tassement s est la somme de deux tassements $s_{1}$ et $s^{\prime}{ }_{1}$ défini pour $s_{1}$ par :

$$
\frac{d s_{1}}{k d z}=\ln \frac{q+\sigma_{0}^{\prime}+\gamma^{\prime} z}{\sigma_{c}^{\prime}+\sigma_{0}^{\prime}+\gamma^{\prime} z}
$$

qui conduit au tassement de compression

$$
\frac{\mathrm{s}_{1}}{\mathrm{kH}}=\mathbf{L}\left(\mathrm{u}, \mathrm{v}^{\prime}\right)
$$




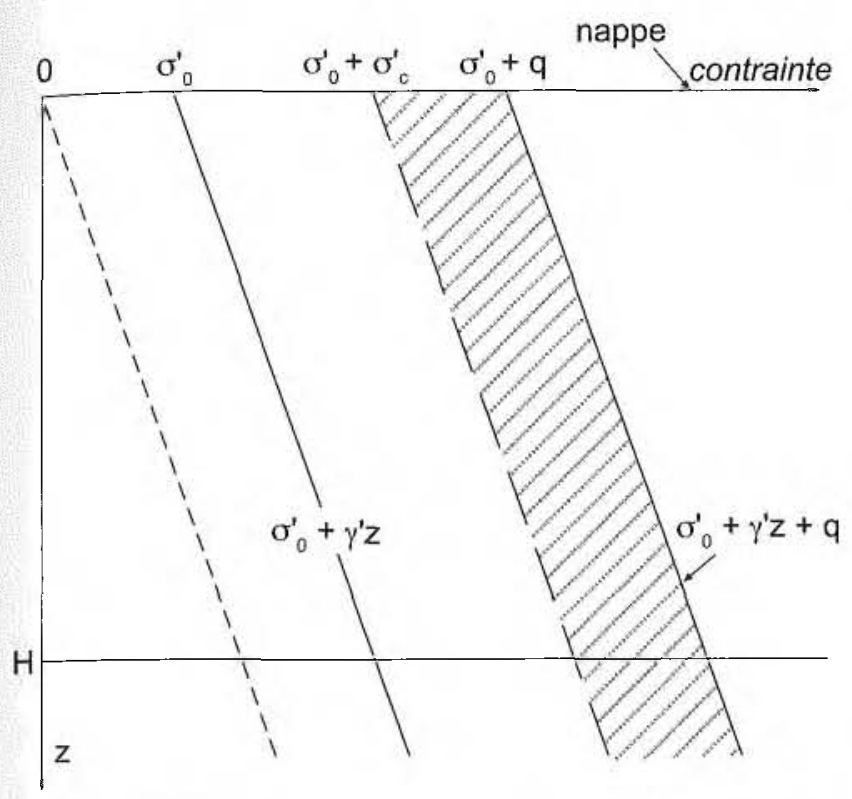

FIG. 3 Sol surconsolidé, chargé et surchargé par q.

Overconsolidated loaded soil, with surcharge q.

et de même au tassement de recompression, lié au rechargement jusqu'à $\sigma_{c}^{\prime}$

$$
\frac{\mathrm{s}_{1}^{\prime}}{\mathrm{k}^{\prime} \mathrm{H}}=\mathrm{L}\left(\mathrm{v}^{\prime}, \mathrm{v}\right)
$$

avec $u=\frac{q+\sigma_{0}^{\prime}}{\gamma^{\prime} H}, v^{\prime}=\frac{\sigma_{c}^{\prime}+\sigma_{0}^{\prime}}{\gamma^{\prime} H}$ et $v=\frac{\sigma_{0}^{\prime}}{\gamma^{\prime} H}$

Si $q<\sigma^{\prime}$; il y a uniquement rechargement, et le tassement par recompression vaut simplement:

$$
\frac{\mathrm{s}^{t}}{\mathrm{k}^{\prime} \mathrm{H}}=\mathrm{L}(\mathrm{u}, \mathrm{v})
$$

On tire de ces trois expressions, celles correspondant à $\sigma_{0}^{\prime}=0$, pour laquelle $\mathrm{V}=0$ et $\mathrm{V}^{\mathrm{v}}=1$.

Si l'on souhaite calculer les tassements de la tranche de sol entre 0 et $\mathrm{z}<\mathrm{H}$, on applique les mêmes formules, mais en remplaçant 1 par $\mathrm{z} / \mathrm{H}, \mathrm{u}, \mathrm{v}^{\prime}$ et $\mathrm{v}$ étant toujours définis par rapport à $\mathrm{H}$, et donc inchangés. On retrouve alors les expressions (6) et (7).

\section{7}

\section{Couche de sol normalement consolidée, saturée et objet d'un rabattement de nappe}

La nappe a toujours été au niveau 0 . On suppose maintenant qu'elle est abaissée pour raison de travaux par exemple.

Le rabattement est supposé être suffisamment long pour entraîner la consolidation complète des couches compressibles et les tassements associés. L'épaisseur globale $\mathrm{H}$ est soumise à ce rabattement sur une hauteur limitée $h \leq \mathrm{H}$.

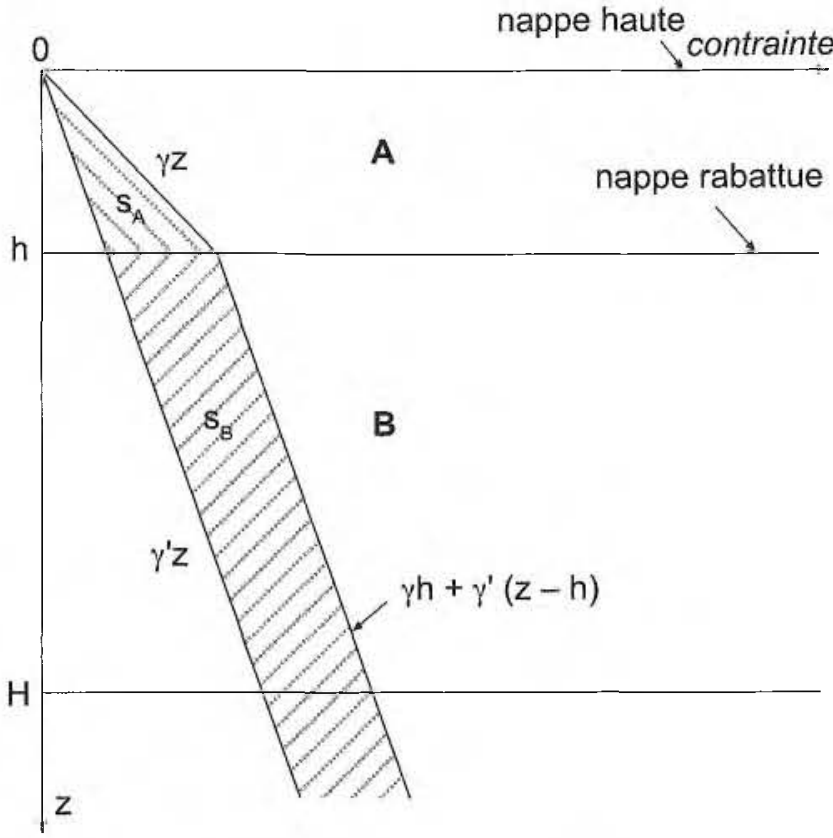

F16. 4 Sol normalement consolidé et saturé, avec rabattement.

Normally consolidated saturated soil, with lowering water table.

Vis-à-vis des tassements provoqués par ce rabattement, il existe deux zones du massif, qui sont à distinguer : entre 0 et $h$, où la contrainte engendrée est linéaire avec $\mathrm{z}$ et vaut $\left(\gamma-\gamma^{\prime}\right) \mathrm{z}$, et entre h et $\mathrm{H}$, où cette contrainte est constante, et vaut $\left(\gamma-\gamma^{\prime}\right)$ h. Le poids volumique $\gamma$ à prendre en compte est celui non saturé de la tranche de sol $(0$ à h) après baisse de la nappe. A défaut de connaître précisément cette valeur, on peut faire l'hypothèse que $\gamma=\gamma_{w}+\gamma^{\prime}$ ce qui revient à dire que l'on ne désature pratiquement pas le sol. C'est légèrement sécuritaire, dans le cas de figure étudié ici, puisque cela conduit à calculer un tassement un peu plus fort que la réalité.

Pour la zone $\mathrm{A}$, entre 0 et $\mathrm{h}$, on écrit :

$$
\frac{d s_{A}}{k d z}=\ln \frac{\left(\gamma^{\prime}+\gamma_{w}\right) z}{\gamma^{\prime} z}
$$

d'où

$$
\frac{\mathrm{s}_{\mathrm{A}}}{\mathrm{kh}}=\ln \frac{\gamma}{\gamma^{\prime}}
$$

Pour la zone B, on peut, avec un changement d'origine des $z_{r}$ calculer son tassement en la considérant comme une couche d'épaisseur $\mathrm{H}$ - h. Cette couche est chargée en tête par $\gamma^{\prime} h$, et surchargée par $\gamma_{w} h$. On pourra alors appliquer l'expression (9) avec $u=\frac{\gamma \mathrm{h}}{\gamma^{\prime} \mathrm{H}^{\prime}}$ et $\mathrm{v}=\frac{\gamma^{\prime} \mathrm{h}}{\gamma^{\prime} \mathrm{H}^{\prime}}$ et donnant: $\frac{\mathrm{s}_{\mathrm{B}}}{\mathrm{kH}}=\mathrm{L}(\mathrm{u}, \mathrm{v})$

Si la nappe, après rabattement, retrouve son niveau 0 d'origine, puis est l'objet de mouvements d'amplitude $h$, le massif peut être considéré comme l'objet de gonflements et recompressions $\mathrm{s}_{\mathrm{A}}$ et $\mathrm{s}_{\mathrm{B}^{\prime}}$ donnés par (14) et (15), mais où $\mathrm{k}^{\prime}$ remplace $\mathrm{k}$. 
Couche de sol saturée, normalement consolidée, chargée en tête et objet d'un rabattement de nappe

Comme dans le cas précédent, deux zones $A$ et $B$ sont à distinguer.

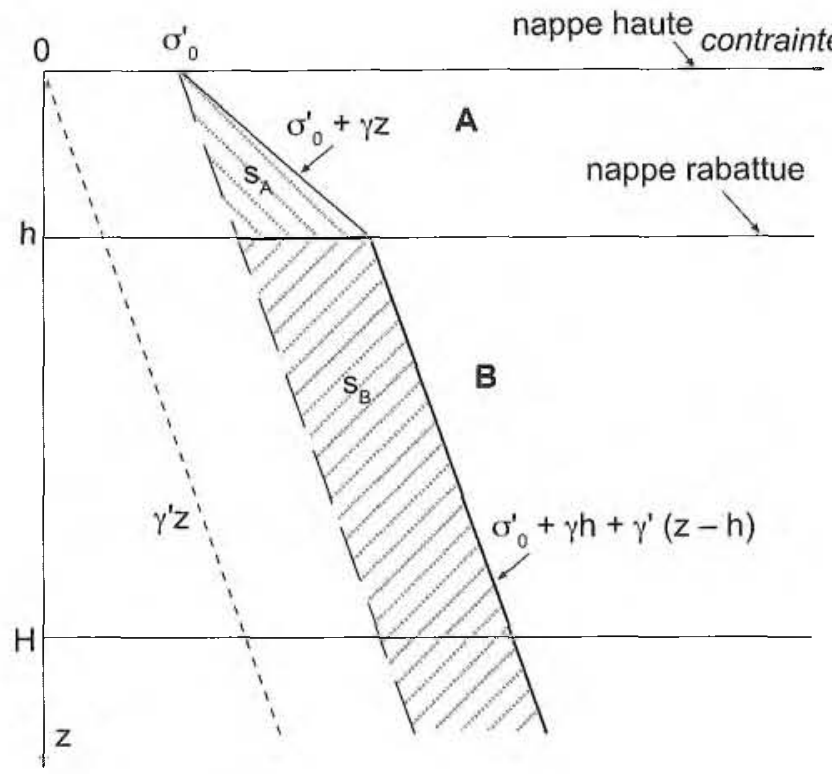

fIG. 5 Sol saturé normalement consolidé, chargé, avec rabattement.

Normally consolidated saturated and loaded soil, with lowering water table.

On montre pour la zone A qu'on peut écrire en

fonction du seul paramètre $v=\frac{\sigma_{0}^{\prime}}{\gamma^{\prime} h}$ :

$$
\frac{\mathrm{s}_{\mathrm{A}}}{\mathrm{kh}}=\mathrm{L}\left(\mathrm{v} \frac{\gamma^{\prime}}{\gamma}, \mathrm{v}, \frac{\gamma}{\gamma^{\prime}}\right)
$$

Si $\sigma_{0}^{\prime}=0$, on retrouve le cas de figure 4 précédent.

Pour la zone B, la configuration est celle de la figure 2 ; en changeant l'origine des $\mathrm{z}$ et en introduisant l'épaisseur $\mathrm{H}^{\prime}=\mathrm{H}-\mathrm{h}$, on obtient :

$$
\frac{\mathrm{s}_{\mathrm{B}}}{\mathrm{k}(\mathrm{H}-\mathrm{h})}=\mathrm{L}(\mathrm{u}, \mathrm{v})
$$

avec $u=\frac{\left(\sigma_{0}^{\prime}+\gamma h\right)}{\gamma^{\prime} H^{\prime}}$ et $v=\frac{\left(\sigma_{0}^{\prime}+\gamma^{\prime} h\right)}{\gamma^{\prime} H^{\prime}}$.

Les remarques précédentes sur les fluctuations de la nappe sont applicables pour estimer les amplitudes $\mathrm{s}_{\mathrm{A}}$ et $\mathrm{s}_{\mathrm{B}}$ des déformations des zones $\mathrm{A}$ et $\mathrm{B}$.

\section{Couche H de sol, saturée, chargée, surchargée en tête et surconsolidée du fait d'une baisse périodique de la nappe}

On ne s'intéresse ici qu'à la seule couche d'épaisseur $h<\mathrm{H}$ d'un massif homogène $\mathrm{H}$ plus épais, soumise à des variations de la nappe, sur toute cette épaisseur h. La durée cumulée de la situation en nappe basse est suffisante pour produire les mêmes tassements qu'une nappe basse permanente. Par ailleurs, les variations de la nappe sont considérées être antérieures à l'application de la charge $q$.

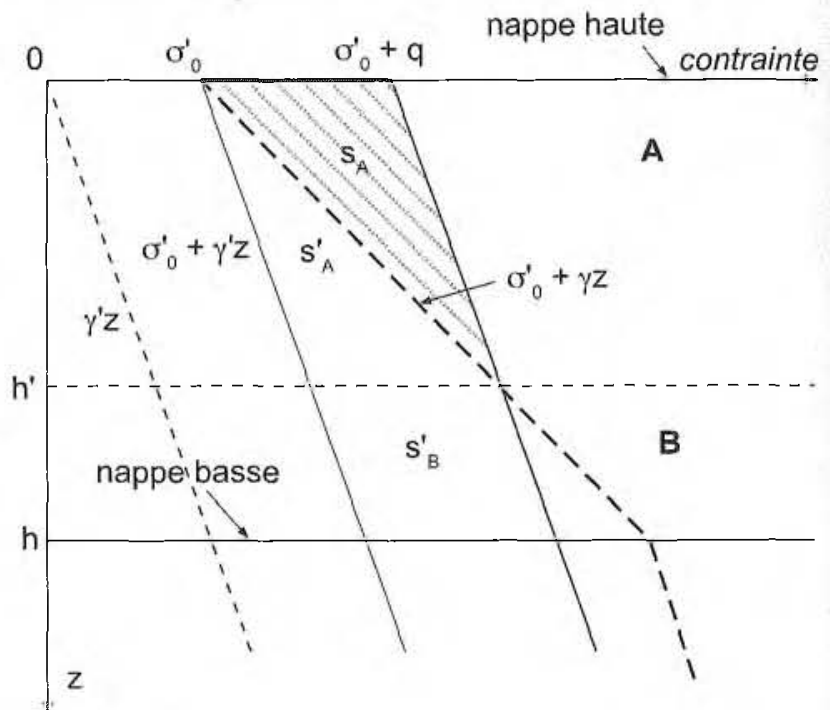

FIG. 6 Sol saturé chargé, avec baisse périodique de la nappe, et surchargé.

Saturated loaded soil, with episodic dewatering, with surcharge.

Le calcul est effectué en position de nappe haute, au niveau (). Le tassement élémentaire de compression est :

$$
\frac{d s}{k d z}=\ln \frac{q+\sigma_{0}^{\prime}+\gamma^{\prime} z}{\sigma_{0}^{\prime}+\gamma^{\prime} z+\gamma_{w} z} \text { tant que } q>\gamma_{w} z \text {. }
$$

Si $q>\gamma_{w} h$, l'intégration est faite sur toute la hauteur h.

Si $q<\gamma, h$, on peut définir une zone $A$ d'épaisseur $h^{\prime}<h$, telle que $q=\gamma_{v} h^{\prime}$, l'intégration portant alors sur la hauteur h' de la zone $\mathrm{A}$.

On obtient pour la zone $A$, avec $u=\frac{q+\sigma_{0}^{\prime}}{\gamma^{\prime} h^{\prime}}$ et $\mathrm{v}=\frac{\sigma_{0}^{\prime}}{\gamma \mathrm{h}^{\prime}}$ un tassement de compression :

$$
\frac{\mathrm{s}_{\mathrm{A}}}{\mathrm{kh}^{\prime}}=\mathrm{L}\left(\mathrm{u}, \mathrm{v}, \frac{\gamma^{\prime}}{\gamma}\right)
$$

auquel on ajoute un tassement de recompression $s^{\prime}$ tel que $\frac{\mathrm{ds}_{\mathrm{A}}^{\prime}}{\mathrm{k}^{\prime} \mathrm{dz}}=\ln \frac{\sigma_{0}^{\prime}+\gamma \mathrm{z}}{\sigma_{0}^{\prime}+\gamma^{\prime} \mathrm{z}}$, soit : 


$$
\frac{\mathrm{s}_{\mathrm{A}}^{\prime} \mathrm{A}}{\mathrm{k}^{\prime} \mathrm{h}^{\prime}}=\mathrm{L}\left(\mathrm{v}, \mathrm{v} \frac{\gamma}{\gamma^{\prime}}, \frac{\gamma}{\gamma^{\prime}}\right)
$$

Pour la zone B d'épaisseur h-h', le tassement s' est donné par:

$$
\frac{\mathrm{s}_{\mathrm{B}}^{\prime}}{\mathrm{k}^{\prime}\left(\mathrm{h}-\mathrm{h}^{\prime}\right)}=\mathrm{L}(\mathrm{u}, \mathrm{v})
$$

avec $u=\frac{q+\sigma_{0}^{\prime}+\gamma^{\prime} h^{\prime}}{\gamma^{\prime}\left(h-h^{\prime}\right)}$ et $v=\frac{\sigma_{0}^{\prime}+\gamma^{\prime} h^{\prime}}{\gamma^{\prime}\left(h-h^{\prime}\right)}$.

Pour le sol situé au-delà de la profondeur h, le problème est celui de la figure 3 où la contrainte de surconsolidation est $\sigma_{c}^{\prime}=\gamma_{w}$ h. Avec un changement d'origine des $z$, on applique les expressions (11), (12) ou (13) en fonction des valeurs relatives de $q$ et $\gamma_{w}$ h.

Si $\sigma_{0}^{\prime}=0$, les formules se simplifient.

\section{0}

\section{Amélioration d'un sol compressible par groupe d'inclusions rigides}

Ce principe de construction d'ouvrages de grandes dimensions sur sols mous, schématisé sur la figure 7 , consiste à fortement réduire la surcharge apportée (remblai, radier souple, dallage) par la mise en œuvre d'éléments rigides verticaux reportant en très grande partie cette surcharge sur des niveaux de sol résistants et peu déformables. La disposition d'un organe de transition entre l'ouvrage et les têtes d'inclusions, qui est le plus souvent un matelas sablo-graveleux de bonne qualité, favorise le transfert des efforts.

Par ce dispositif, la surface du sol se trouve finalement peu surchargée sous une contrainte résiduelle ; l'effet bénéfique provoqué en tête se poursuit cependant au sein du sol compressible, qui tasse sous cette contrainte résiduelle. Les inclusions sont donc soumises au frottement négatif, ce qui décharge le sol et diminue encore la contrainte verticale induite en profondeur. Il existe donc, le plus souvent, (c un point neutre » au contact de l'inclusion, à une profondeur $\mathrm{z}=\mathrm{h}_{\mathrm{c}^{\prime}}$ où cette contrainte induite est nulle. Du fait du phénomène d'accrochage le long du fût de l'inclusion, il persiste cependant, dans le massif à ce niveau $h$, une contrainte induite moyenne, généralement très faible, appliquée au massif sur toute la surface d'influence de l'inclusion.
En résumé, lorsqu'un groupe d'inclusions est correctement dimensionné, la surcharge q appliquée par l'ouvrage, entraîne à la surface du sol une surcharge résiduelle $q^{\prime}$, valant de 15 à $10 \%$ de q, puis, au niveau du point neutre, une surcharge q" valant de 10 à $5 \%$ de q. C'est ce jeu de contraintes qui détermine le tassement sous l'ouvrage après traitement du massif par les inclusions. Pour ce calcul, on peut, et l'erreur est négligeable, admettre que la variation de q' à q", sur l'épaisseur h. est linéaire. Par ailleurs, les faibles valeurs de q' et $\mathrm{q}^{\prime \prime}$, auxquelles on cherche volontairement à parvenir, montrent, de ce fait, l'importance et donc la nécessité de savoir si le massif est surconsolidé, ne serait-ce que légèrement. Aussi, la variation du niveau de la nappe, entre le printemps et la fin de l'été par exemple, peut générer de telles contraintes de surconsolidation, qui peuvent éventuellement être supérieures aux contraintes résiduelles q' et q". La surconsolidation par dessiccation de couches superficielles peut augmenter, en termes de surconsolidation, l'effet du seul phénomène de battement de la nappe. Les surcharges q' et q" peuvent être déterminées par la méthode développée pour le calcul des inclusions rigides (Combarieu, 1988) ou mieux par cette même méthode améliorée (Combarieu, 2008). Sont fournies, ci-après, les expressions des tassements pour plusieurs cas de figures qui peuvent être rencontrés.

\section{1}

\section{Couche normalement consolidée, surchargée par le diagramme q'-q", nappe en surface, invariable}

La contrainte due à la surcharge est $q(z)=q^{\prime}-a z$ avec $a=\frac{q^{\prime}-q^{\prime \prime}}{h_{c}}$, ce qui conduit aux expressions suivan-
tes.

Pour la zone $\mathrm{A}$, de 0 à h :

$$
\frac{\mathrm{s}_{\mathrm{A}}}{\mathrm{kh}_{\mathrm{c}}}=\mathrm{L}\left(\mathrm{u}, 0, \frac{\gamma^{\prime}-\mathrm{a}}{\gamma^{\prime}}\right)
$$

en posant $u=\frac{q^{\prime}}{\left(\gamma^{\prime}-a\right) h_{c}}$.
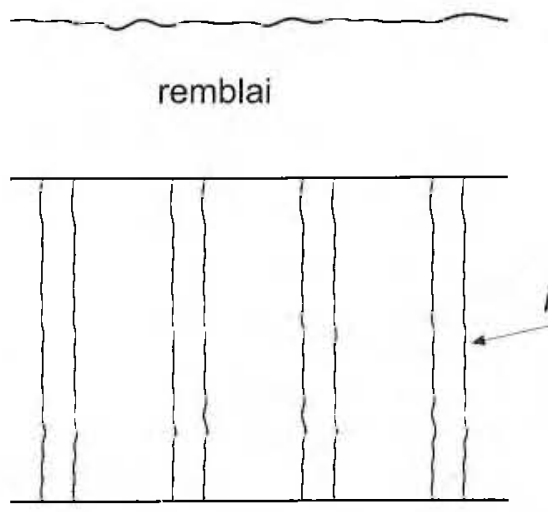

FIG. 7

Principe de renforcement par inclusions rigides. Soil reinforcement by rigid inclusions.

\section{charge $\mathrm{q}$}

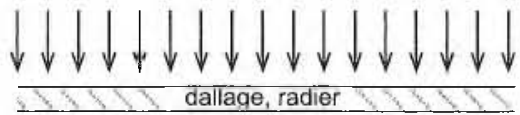

matelas de graves

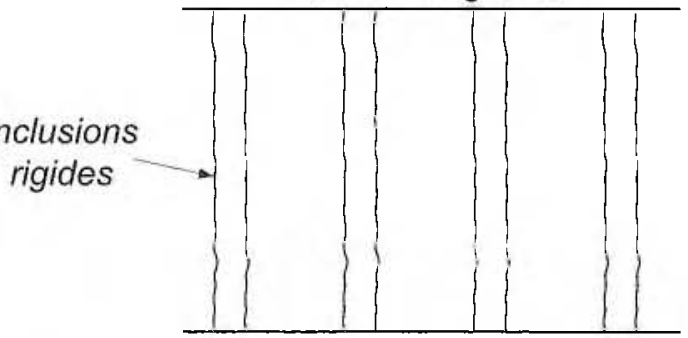




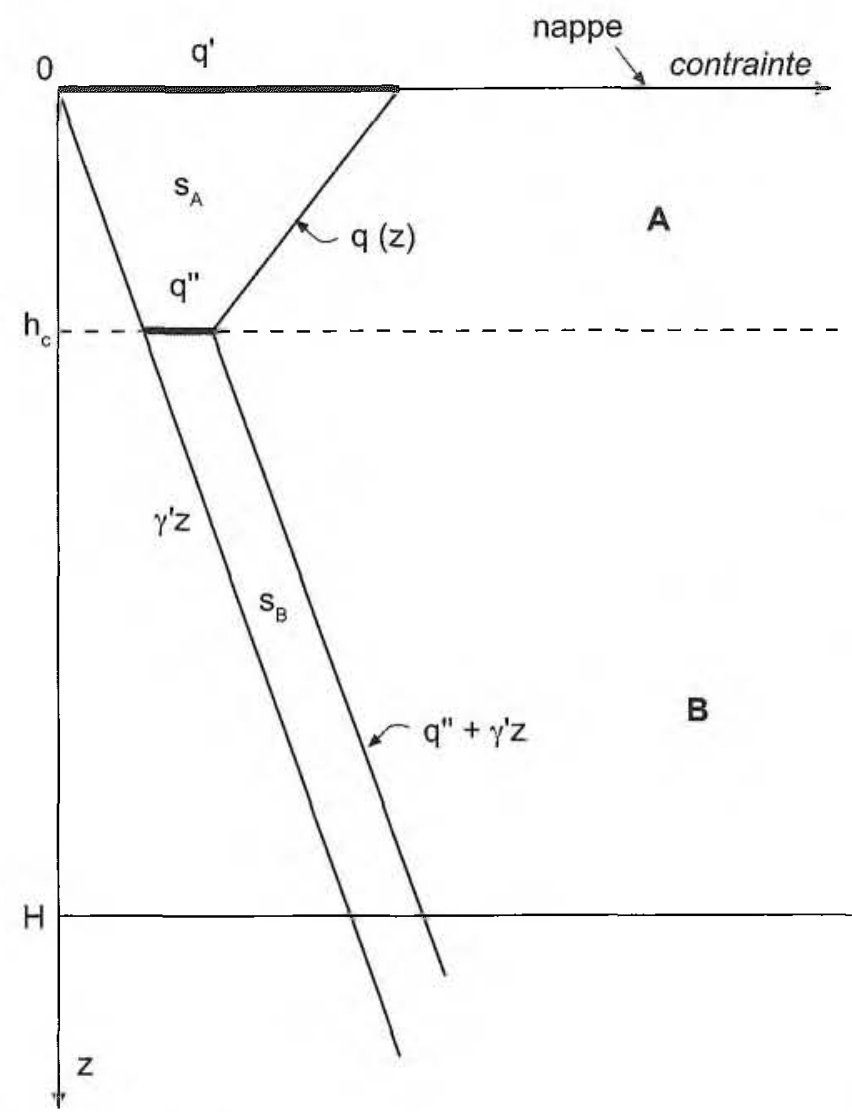

FIG. 8 Sol saturé normalement consolidé surchargé par $\mathbf{q}(\mathrm{z})$.

Normally consolidated saturated soil, with surcharge $\mathrm{g}(\mathrm{z})$

Pour la zone $\mathrm{B}$, de $\mathrm{h}_{\mathrm{c}}$ à $\mathrm{H}$, le sol est soumis en tête à la charge $\gamma^{\prime} h_{c}$ et à la surcharge $q^{\prime \prime}$. On obtient donc, comme pour la relation (9):

$$
\frac{\mathrm{s}_{\mathrm{B}}}{\mathrm{k}\left(\mathrm{H}-\mathrm{h}_{\mathrm{c}}\right)}=\mathrm{L}(\mathrm{u}, \mathrm{v})
$$

avec $u=\frac{q^{\prime \prime}+\gamma^{\prime} h_{c}}{\gamma^{\prime}\left(H-h_{c}\right)}$ et $v=\frac{\gamma^{\prime} h_{c}}{\gamma^{\prime}\left(H-h_{c}\right)}$

\section{2}

\section{Couche surconsolidée sous $\sigma^{\prime \prime}$ surchargée par le diagramme $q^{\prime}-q^{\prime \prime}$ sur une hauteur $h_{c}$ i nappe en surface invariable}

Il est important de pouvoir analyser précisément cette situation géotechnique. Pour le renforcement par inclusions rigides, la confrontation entre $\sigma_{c}^{\prime}$ et les valeurs du diagramme de la surcharge $q(z)$ influe directement sur la densité du renforcement, déterminée par les critères de tassement. Ainsi, si le maillage des inclusions est tel qu'il conduise à une valeur $q^{\prime}$ et, a fortiori, $q^{\prime \prime}$ inférieure ou égale à $\sigma^{\prime}$, le tassement vertical est pratiquement éliminé.

Cette surconsolidation, relativement fréquente dans les sols compressibles, n'interesse généralement qu'une frange supérieure du massif. Son effet bénéfique peut se cumuler avec celui d'une variation de la nappe provoquant également une surconsolidation dans l'ensemble du massif.

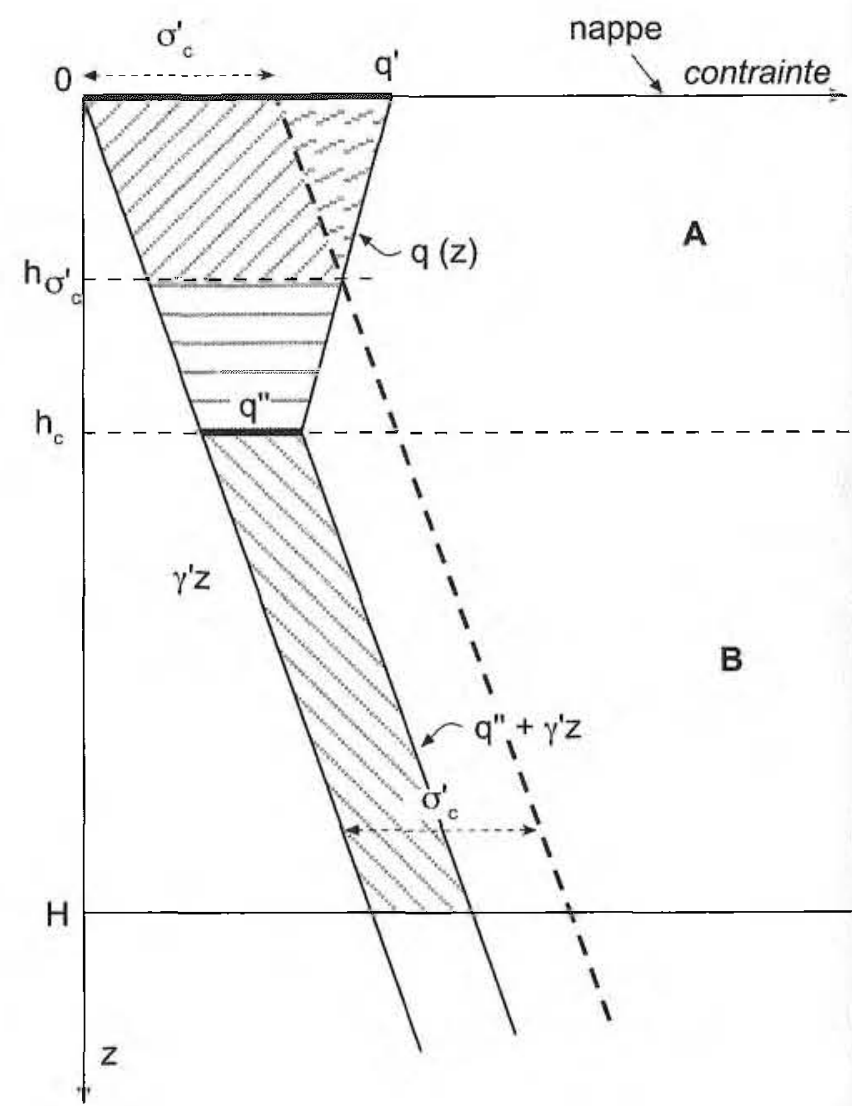

FIG. 9 Sol saturé, surchargé par $q(z)$, et surconsolidé.

Overconsolidated saturated soil, with surcharge $\mathrm{q}(\mathrm{z})$.

Dans cette situation, il y a trois cas à considérer.

- Si $\sigma_{c}^{\prime}>q^{\prime}$, le tassement comporte alors deux termes :

- un tassement de recompression sur l'épaisseur $h_{c}$ :

$$
\frac{\mathrm{s}_{\mathrm{A}}^{\prime}}{\mathrm{k}^{\prime} \mathrm{h}_{\mathrm{C}}}=\mathrm{L}\left(\mathrm{u}, 0, \frac{\gamma^{\prime}-\mathrm{a}}{\gamma^{\prime}}\right)
$$

avec $u=\frac{q^{\prime}}{\left(\gamma^{\prime}-a\right) h_{c}}$;

- un tassement de recompression sur l'épaisseur $\mathrm{H}-\mathrm{h}_{\mathrm{c}}$ :

$$
\frac{\mathrm{s}_{\mathrm{B}}^{\prime}}{\mathrm{k}^{\prime}\left(\mathrm{H}-\mathrm{h}_{\mathrm{c}}\right)}=\mathrm{L}(\mathrm{u}, \mathrm{v})
$$

avec $u=\frac{q^{\prime \prime}+\gamma^{\prime} h_{c}}{\gamma^{\prime}\left(H-h_{c}\right)}$ et $v=\frac{\gamma^{\prime} h_{c}}{\gamma^{\prime}\left(H-h_{c}\right)}$.

- Si $q^{\prime}>\sigma^{\prime}>q^{\prime \prime}$, cette configuration détermine une profondeur $h_{\sigma_{c}^{\prime}}$ telle que $h_{\sigma_{c}^{\prime}}=\frac{q^{\prime}-\sigma_{c}^{\prime}}{a}$, cela conduit aux tassements suivants :

- entre 0 et $h_{\sigma^{\prime}}$, un tassement de compression

$$
\frac{\mathrm{s}}{\mathrm{kh}_{\mathrm{o}_{\mathrm{c}}^{\prime}}}=\mathrm{L}\left(\mathrm{u}_{1}, \mathrm{x}, \frac{\gamma^{\prime}-\mathrm{a}}{\gamma^{\prime}}\right)
$$


et un tassement de recompression

$$
\frac{\mathrm{s}^{\prime}}{\mathrm{k}^{\prime} \mathrm{h}_{\mathrm{\sigma}_{\mathrm{c}}^{\prime}}}=\mathrm{L}(\mathrm{x}, 0)
$$

$\operatorname{avec} u_{1}=\frac{q^{\prime}}{\left(\gamma^{\prime}-a\right) h_{\sigma_{c}^{\prime}}}$ et $x=\frac{\sigma_{c}^{\prime}}{\left(\gamma^{\prime}-a\right) h_{\sigma_{c}^{\prime}}}$

- entre $h_{\sigma_{c_{c}^{\prime}}}$ et $h_{c^{\prime}}$ un tassement de recompression

$$
\frac{\mathrm{s}^{\prime}}{\mathrm{k}^{\prime}\left(\mathrm{h}_{\mathrm{c}}-\mathrm{h}_{\sigma_{\mathrm{c}}^{\prime}}\right)}=\mathrm{L}\left(\mathrm{x}^{\prime}, \mathrm{v}^{\prime}, \frac{\gamma^{\prime}-\mathrm{a}}{\gamma}\right)
$$

avec $v^{\prime}=\frac{\gamma^{\prime} h_{\sigma_{C}^{\prime}}}{\gamma^{\prime}\left(h_{c}-h_{\sigma_{c}^{\prime}}\right)}$ et $x^{\prime}=\frac{\sigma_{c}^{\prime}+\gamma^{\prime} h_{\sigma_{c}^{\prime}}}{\gamma^{\prime}\left(h_{c}-h_{\sigma_{c}^{\prime}}\right)}$;

- enfin, entre $h_{c}$ et $H$, un tassement de recompression

$$
\frac{\mathrm{s}^{\prime}}{\mathrm{k}^{\prime}\left(\mathrm{H}-\mathrm{h}_{\mathrm{c}}\right)}=\mathbf{L}(\mathrm{u}, \mathrm{v})
$$

avec $u=\frac{q^{\prime \prime}+\gamma^{\prime} h_{c}}{\gamma^{\prime}\left(H-h_{c}\right)}$ et $v=\frac{\gamma^{\prime} h_{c}}{\gamma^{\prime}\left(H-h_{c}\right)}$.

- Si $\mathrm{q}^{\prime \prime}>\sigma_{c}^{\prime}$ :

- entre 0 et $h_{c}$, on a un tassement de compression

$$
\frac{\mathrm{s}}{\mathrm{kh}} \mathrm{c}=\mathrm{L}\left(\mathrm{u}, \mathrm{x}, \frac{\gamma^{\prime}-\mathrm{a}}{\gamma^{\prime}}\right)
$$

et un tassement de recompression

$$
\frac{\mathrm{s}^{\prime}}{\mathrm{k}^{\prime} \mathrm{h}_{\mathrm{c}}}=\mathbf{L}(\mathrm{x}, 0)
$$

$\operatorname{avec} x=\frac{\sigma_{c}^{\prime}}{\gamma^{\prime} h_{c}}$ et $u=\frac{q^{\prime}}{\left(\gamma^{\prime}-a\right) h_{c}}$;

- entre $h_{c}$ et $\mathrm{H}$, un tassement de compression

$$
\frac{\mathrm{s}}{\mathrm{k}\left(\mathrm{H}-\mathrm{h}_{\mathrm{c}}\right)}=\mathrm{L}\left(\mathrm{u}, \mathrm{v}^{\prime}\right)
$$

et un tassement de recompression

$$
\frac{\mathrm{s}^{\prime}}{\mathrm{k}^{\prime}\left(\mathrm{H}-\mathrm{h}_{\mathrm{c}}\right)}=\mathrm{L}\left(\mathrm{v}^{\prime}, \mathrm{v}\right)
$$

avec $u=\frac{q^{\prime \prime}+\gamma^{\prime} h_{c}}{\gamma^{\prime}\left(H-h_{c}\right)^{\prime}}, v=\frac{\gamma^{\prime} h_{c}}{\gamma^{\prime}\left(H-h_{c}\right)}$ et $v^{\prime}=\frac{\gamma^{\prime} h_{c}+\sigma_{c}^{\prime}}{\gamma^{\prime}\left(H-h_{c}\right)}$.

\section{Couche chargée en tête, surchargée sous le diagramme q'-q" sur une hauteur $h_{c}$ et surconsolidée sur une hauteur $h$ par une baisse alternée de la nappe}

C'est un cas relativement courant dans le domaine du renforcement par inclusions rigides. La charge $\sigma_{0}^{\prime}$ correspond soit à du matériau mis en œuvre préalablement à la réalisation des inclusions et considéré comme ayant totalement provoqué le tassement du sol compressible (plate-forme de travail par exemple), soit simplement à une couche superficielle de sol naturel hors d'eau.

Six cas de figure différents peuvent théoriquement se présenter, en fonction des valeurs relatives de $h$, $h_{c}$ et $H$, d'une part, et de $q^{\prime}, q^{\prime \prime}$ et $h \gamma_{w^{\prime}}$ d'autre part. Il faut, cependant, remarquer que les tassements de recompression (notés “ prime ») seront, en général, extrêmement faibles, compte tenu de la modicité de la surcharge $q^{\prime \prime}$.

Pour ces six situations, définies sur les figures 10 à 15, les tassements sont calculés pour chacune des couches $\mathrm{A}, \mathrm{B}, \mathrm{C}$, voire $\mathrm{D}$, à partir des trois expressions élémentaires (1), (2) et (3). On donne, sous forme de tableaux, pour chaque figure et chaque niveau, les valeurs de $u, v$ et $B / D$ conformément à l'intégrale $L$ (4) (pour u et v, afin d'alléger les tableaux, on donne leur produit par l'épaisseur correspondante de chacune des couches concernées).

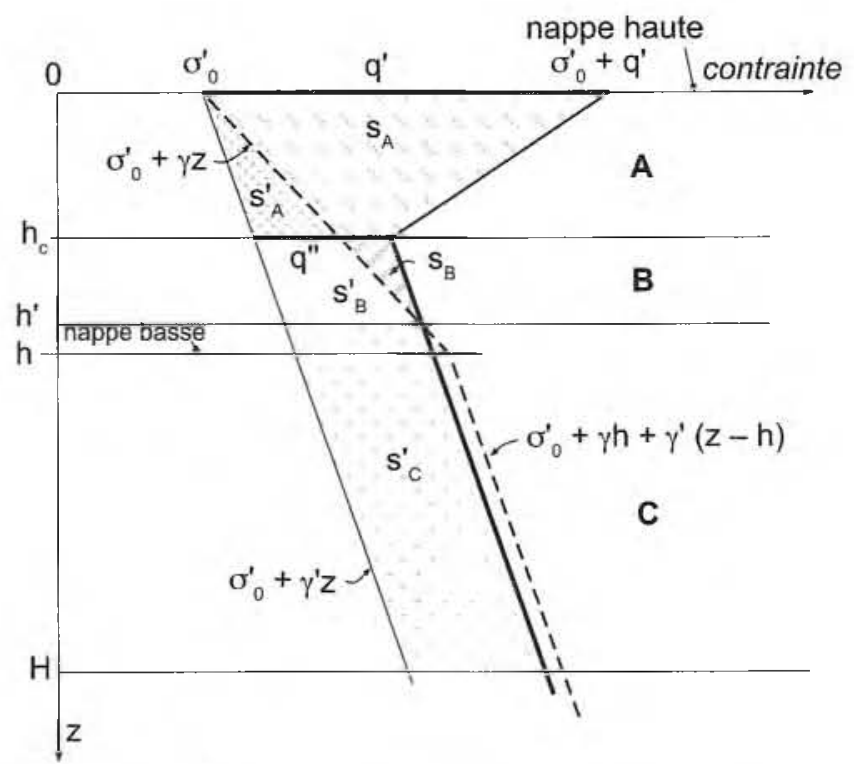

FIG. 10 Sol saturé chargé, surchargé par $q(z)$, avec nappe variable.

Saturated loaded soil, with periodic dewatering and surcharge $q(z)$.
Le cas de la figure 10 est celui d'une valeur de $h_{c}$ pour le point neutre, inférieure à $h$, niveau bas de la nappe et avec $\gamma_{w} h_{c}<q^{\prime \prime}<\gamma_{w} h$; ce qui conduit à une valeur $h^{\prime}$ telle que $\gamma_{w} h^{\prime}=q^{\prime \prime}$ avec $h_{c}<h^{\prime}<h<H$. 
TABLEAU II Expressions des tassements d'après la figure 10.

Expressions for settlements from figure 10.

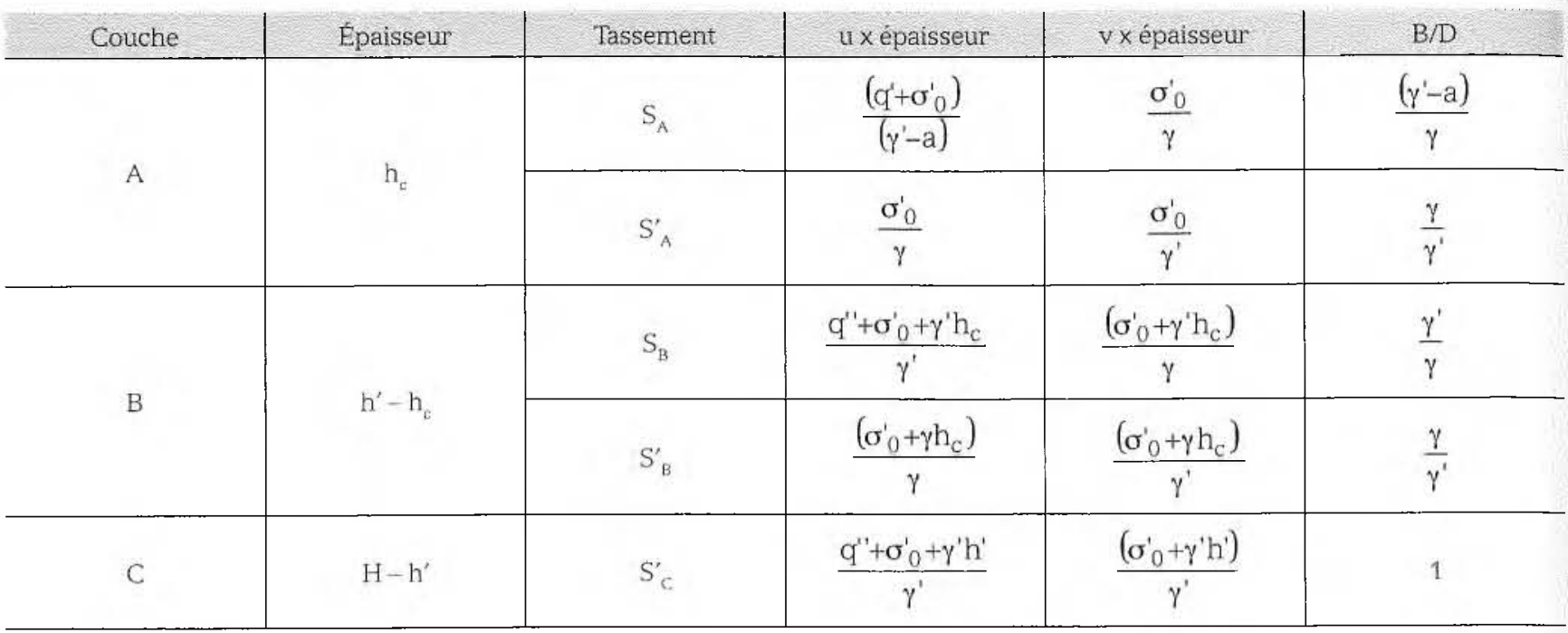

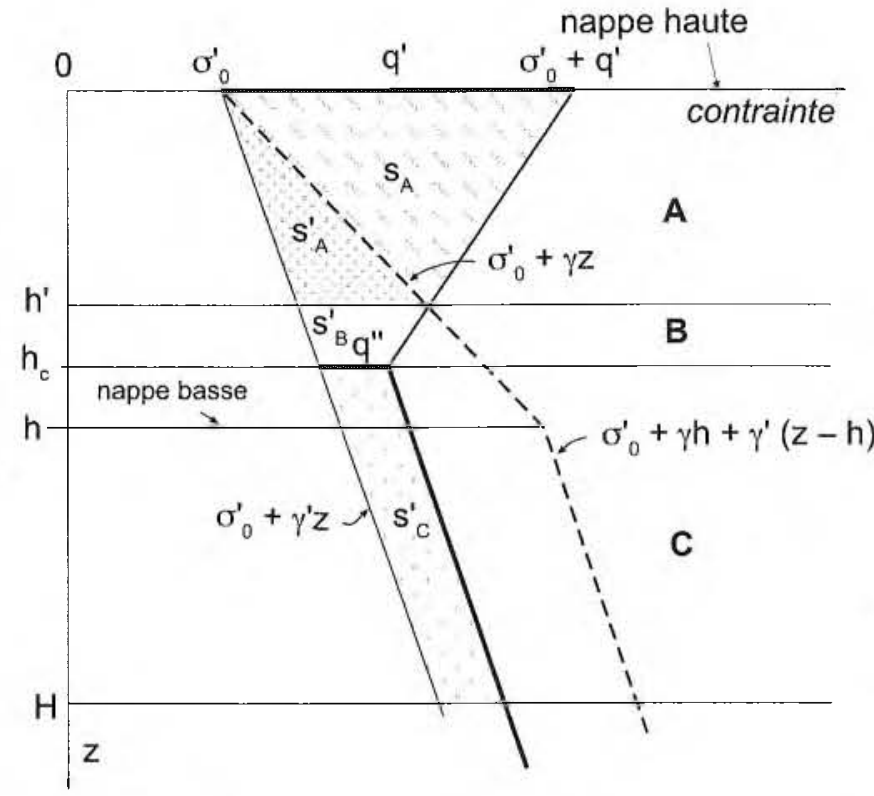

FIG. 11 Sol saturé chargé, surchargé par $\mathrm{q}(\mathrm{z})$, avec nappe variable, $\mathbf{q}^{\prime \prime}<\gamma_{\mathrm{w}} \mathbf{h}^{\prime}$ et $\mathbf{h}^{\prime}<\mathbf{h}_{\mathrm{c}}<\mathbf{h}<\mathrm{H}$. Saturated loaded soil, with periodic dewatering and surcharge $q(z)$.

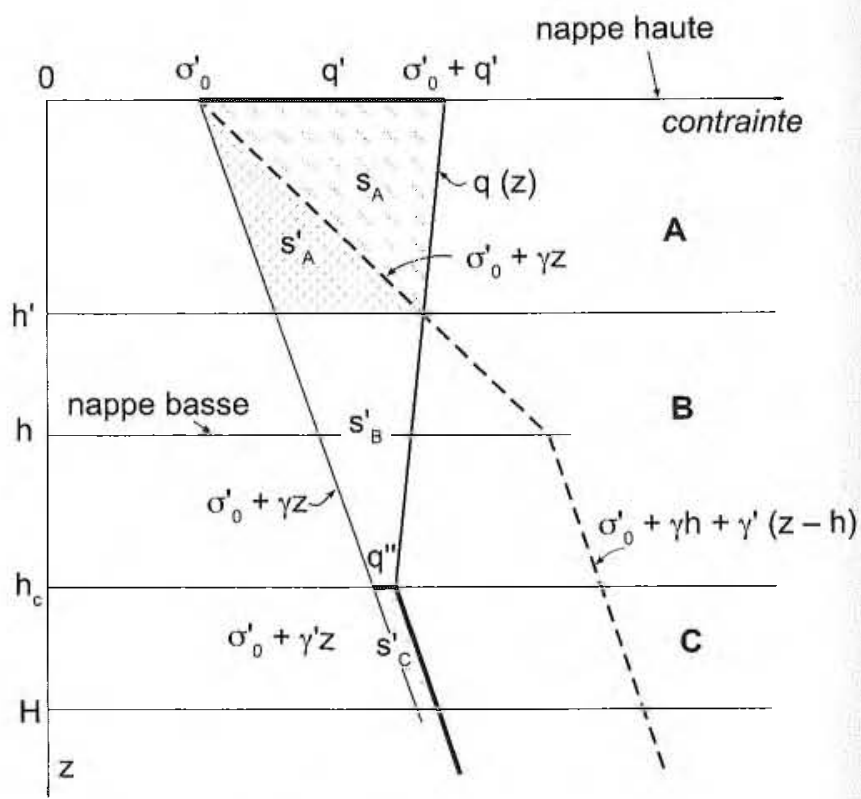

FIG. 12 Sol saturé chargé, surchargé par $q(\mathrm{z})$, avec nappe variable, $\mathbf{h}^{\prime}<\mathbf{h}<\mathbf{h}<H$ et $\mathbf{q}^{\prime \prime}<\gamma_{\omega} \mathbf{h}^{\prime}$. Saturated loaded soil, with periodic dewatering and surcharge $q(z)$. 
tABLEAU II Expressions des tassements d'après les figures 11 et 12. Expressions for settlements from figures 11 and 12.

\begin{tabular}{|c|c|c|c|c|c|}
\hline Couche & Épaisseur & Tassement & $\mathrm{u} \times$ épaisseur & $\mathrm{v} \times$ épaisseur & $B / D$ \\
\hline \multirow{2}{*}{ A } & \multirow{2}{*}{$h^{\prime}$} & $\mathrm{S}_{\mathrm{A}}$ & $\frac{\left(q^{\prime}+\sigma_{0}^{\prime}\right)}{\left(\gamma^{\prime}-a\right)}$ & $\frac{\sigma_{0}^{\prime}}{\gamma}$ & $\frac{\left(\gamma^{\prime}-\mathrm{a}\right)}{\gamma}$ \\
\hline & & $\mathrm{S}_{\mathrm{A}}^{\prime}$ & $\frac{\sigma_{0}^{\prime}}{\gamma}$ & $\frac{\sigma_{0}^{\prime}}{\gamma^{\prime}}$ & $\frac{\gamma}{\gamma^{\prime}}$ \\
\hline B & $h_{c}-h^{\prime}$ & $\mathrm{S}_{\mathrm{B}}^{\prime}$ & $\frac{q^{\prime}+\sigma_{0}^{\prime}+\left(\gamma^{\prime}-a\right) h^{\prime}}{\gamma^{\prime}-a}$ & $\frac{\left(\sigma_{0}^{\prime}+\gamma^{\prime} h^{\prime}\right)}{\gamma^{\prime}}$ & $\frac{\left(\gamma^{\prime}-a\right)}{\gamma^{\prime}}$ \\
\hline C & $\mathrm{H}-\mathrm{h}_{\mathrm{c}}$ & $S_{C}^{\prime}$ & $\frac{q^{\prime \prime}+\sigma_{0}^{\prime}+\gamma^{\prime} h_{c}}{\gamma^{\prime}}$ & $\frac{\left(\sigma_{0}^{\prime}+\gamma^{\prime} h_{c}\right)}{\gamma^{\prime}}$ & 1 \\
\hline
\end{tabular}

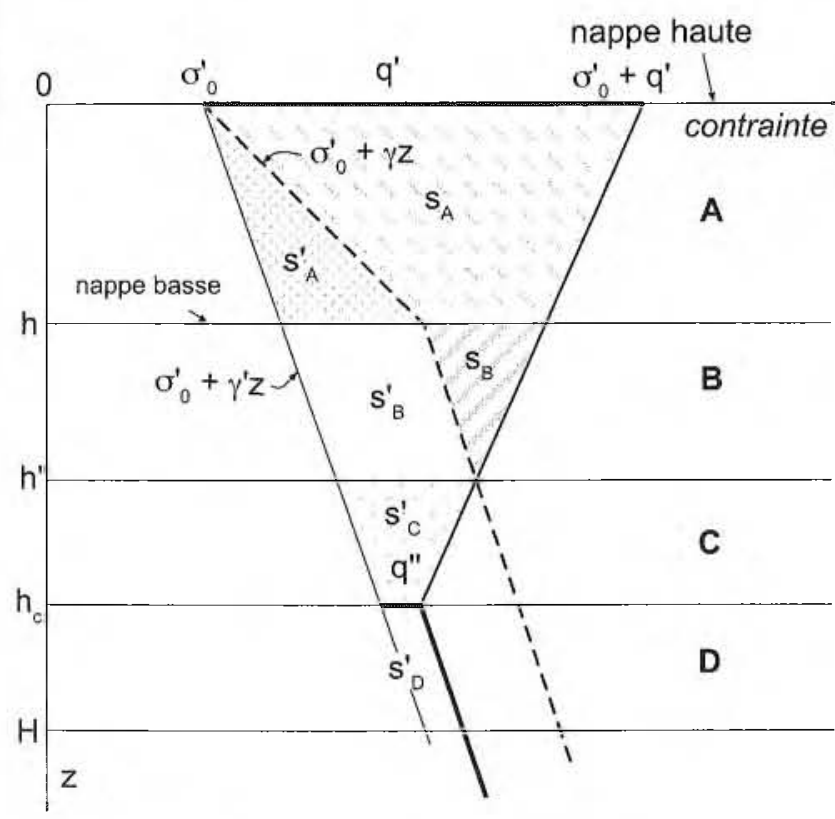

FIG. 13 Sol saturé chargé, surchargé par q(z), et nappe variable $\mathbf{h}<\mathrm{h}^{\prime}<\mathrm{h}_{\mathrm{c}}<\mathrm{H}$.

Saturated loaded soil. with periodic dewatering and surcharge $\mathrm{q}(\mathrm{z})$.

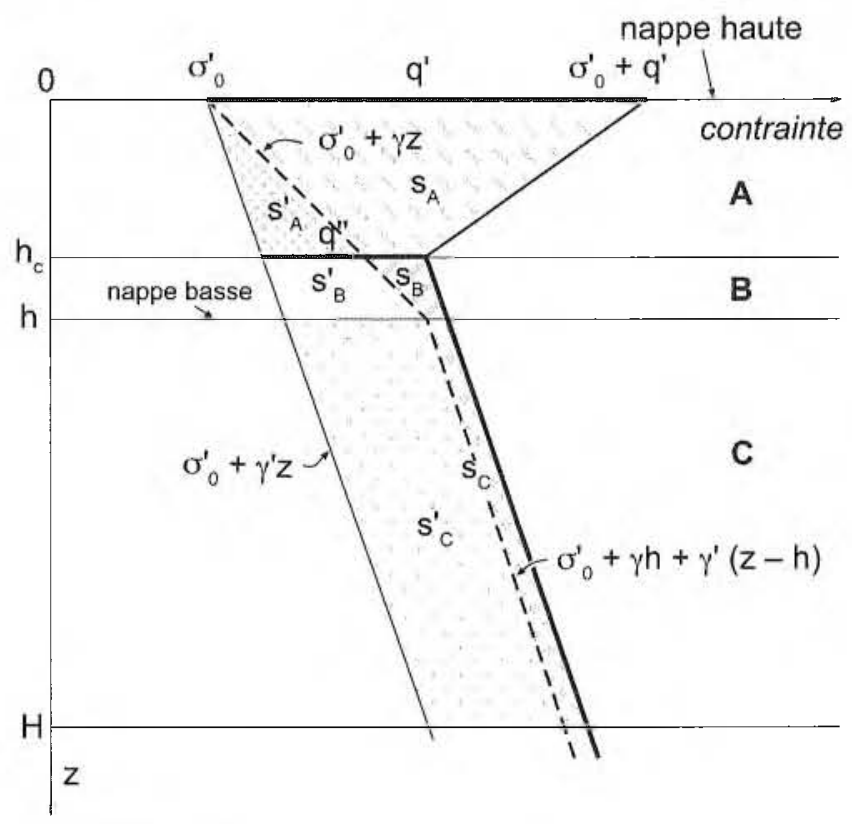

FIG. 14 Sol saturé chargé, surchargé par q(z), et nappe variable $\mathbf{h}_{\mathbf{c}}<\mathbf{h}<\mathrm{H}$.

Saturated loaded soil, with periodic dewatering and surcharge $q(z)$. 
Expressions des tassements d'après la figure 13.

Expressions for settlements from figure 13.

\begin{tabular}{|c|c|c|c|c|c|}
\hline Couche & Épaisseur & Tassement & u xépaisseur & $\mathrm{V} \times$ épaisseur & $\mathrm{B} / \mathrm{D}$ \\
\hline \multirow{2}{*}{ A } & \multirow{2}{*}{$\mathrm{h}$} & $\mathrm{S}_{\mathrm{A}}$ & $\frac{\left(q^{\prime}+\sigma_{0}^{\prime}\right)}{\left(\gamma^{\prime}-a\right)}$ & $\frac{\sigma_{0}^{\prime}}{\gamma}$ & $\frac{\left(\gamma^{\prime}-\mathrm{a}\right)}{\gamma}$ \\
\hline & & $\mathrm{S}_{\mathrm{A}}^{\prime}$ & $\frac{\sigma_{0}^{\prime}}{\gamma}$ & $\frac{\sigma_{0}^{\prime}}{\gamma^{\prime}}$ & $\frac{\gamma}{\gamma^{\prime}}$ \\
\hline \multirow{2}{*}{ B } & \multirow{2}{*}{$h^{\prime}-h$} & $\mathrm{~S}_{\mathrm{B}}$ & $\frac{\mathrm{q}^{\prime}+\sigma_{0}^{\prime}+\left(\gamma^{\prime}-\mathrm{a}\right) \mathrm{h}}{\gamma^{\prime}-\mathrm{a}}$ & $\frac{\left(\sigma_{0}^{\prime}+\gamma h\right)}{\gamma^{\prime}}$ & $\frac{\gamma^{\prime}-\mathrm{a}}{\gamma^{\prime}}$ \\
\hline & & $S_{B}^{\prime}$ & $\frac{\left(\sigma_{0}^{\prime}+\gamma h\right)}{\gamma^{\prime}}$ & $\frac{\left(\sigma_{0}^{\prime}+\gamma^{\prime} h\right)}{\gamma^{\prime}}$ & 1 \\
\hline C & $\mathrm{h}_{c}-\mathrm{h}^{\prime}$ & $S_{C}^{\prime}$ & $\frac{\mathrm{q}^{\prime}+\sigma_{0}^{\prime}+\left(\gamma^{\prime}-\mathrm{a}\right) \mathrm{h}^{\prime}}{\gamma^{\prime}-\mathrm{a}}$ & $\frac{\left(\sigma_{0}^{\prime}+\gamma^{\prime} h^{\prime}\right)}{\gamma^{\prime}}$ & $\frac{\gamma^{\prime}-\mathrm{a}}{\gamma^{\prime}}$ \\
\hline D & $\mathrm{H}-\mathrm{h}_{\mathrm{c}}$ & $S_{D}^{\prime}$ & $\frac{\mathrm{q}^{\prime \prime}+\sigma_{0}^{\prime}+\gamma^{\prime} h_{\mathrm{c}}}{\gamma^{\prime}}$ & $\frac{\left(\sigma_{0}^{\prime}+\gamma^{\prime} h_{c}\right)}{\gamma^{\prime}}$ & 1 \\
\hline
\end{tabular}

TABLEAUV Expressions des tassements d'après la figure 14.

Expressions for settlements from figure 14

\begin{tabular}{|c|c|c|c|c|c|}
\hline Couche & Épaisseur & Tassement & u épaisseur & v épaisseur & $\mathrm{B} / \mathrm{D}$ \\
\hline \multirow{2}{*}{ A } & \multirow{2}{*}{$\mathrm{h}_{c}$} & $\mathrm{~S}_{\mathrm{A}}$ & $\frac{\left(q^{\prime}+\sigma_{0}^{\prime}\right)}{\left(\gamma^{\prime}-a\right)}$ & $\frac{\sigma_{0}^{\prime}}{\gamma}$ & $\frac{\left(\gamma^{\prime}-\mathrm{a}\right)}{\gamma}$ \\
\hline & & $S_{A}^{\prime}$ & $\frac{\sigma_{0}^{\prime}}{\gamma}$ & $\frac{\sigma_{0}^{\prime}}{\gamma^{\prime}}$ & $\frac{\gamma}{\gamma^{\prime}}$ \\
\hline \multirow{2}{*}{ B } & \multirow{2}{*}{$\mathrm{h}-\mathrm{h}_{\mathrm{c}}$} & $\mathrm{S}_{\mathrm{B}}$ & $\frac{q^{\prime \prime}+\sigma_{0}^{\prime}+\gamma^{\prime} h_{c}}{\gamma^{\prime}}$ & $\frac{\left(\sigma_{0}^{\prime}+\gamma^{\prime} h_{c}\right)}{\gamma}$ & $\frac{\gamma^{\prime}}{\gamma}$ \\
\hline & & $S_{B}^{\prime}$ & $\frac{\left(\sigma_{0}^{\prime}+\gamma^{\prime} h_{c}\right)}{\gamma}$ & $\frac{\left(\sigma_{0}^{\prime}+\gamma^{\prime} h_{c}\right)}{\gamma^{\prime}}$ & $\frac{\gamma}{\gamma^{\prime}}$ \\
\hline \multirow{2}{*}{ C } & \multirow{2}{*}{$\mathrm{H}-\mathrm{h}$} & $\mathrm{S}_{\mathrm{C}}$ & $\frac{q^{\prime \prime}+\sigma_{0}^{\prime}+\gamma^{\prime} h}{\gamma^{\prime}}$ & $\frac{\left(\sigma_{0}^{\prime}+\gamma h\right)}{\gamma^{\prime}}$ & 1 \\
\hline & & $s_{C}^{\prime}$ & $\frac{\left(\sigma_{0}^{\prime}+\gamma h\right)}{\gamma^{\prime}}$ & $\frac{\left(\sigma_{0}^{\prime}+\gamma^{\prime} h\right)}{\gamma^{\prime}}$ & 1 \\
\hline
\end{tabular}


TABLEAU YI Expressions des tassements d'après la figure 15.

Expressions for settlements from figure 15.

\begin{tabular}{|c|c|c|c|c|c|}
\hline Couche & Epaisseur & Tassement & u $x$ épaisseur & v xépaisseur & $\mathrm{B} / \mathrm{D}$ \\
\hline \multirow{2}{*}{ A } & \multirow{2}{*}{$\mathrm{h}_{\mathrm{c}}$} & $\mathrm{S}_{\mathrm{A}}$ & $\frac{\left(\alpha^{\prime}+\sigma_{0}^{\prime}\right)}{\left(\gamma^{\prime}-a\right)}$ & $\frac{\sigma_{0}^{\prime}}{\gamma}$ & $\frac{\left(\gamma^{\prime}-a\right)}{\gamma}$ \\
\hline & & $S_{A}^{\prime}$ & $\frac{\sigma_{0}^{\prime}}{\gamma}$ & $\frac{\sigma_{0}^{\prime}}{\gamma^{\prime}}$ & $\frac{\gamma}{\gamma^{\prime}}$ \\
\hline \multirow{2}{*}{ B } & \multirow{2}{*}{$\mathrm{h}_{\mathrm{c}}-\mathrm{h}$} & $\mathrm{S}_{\mathrm{B}}$ & $\frac{q^{\prime}+\sigma_{0}^{\prime}+\left(\gamma^{\prime}-a\right) h}{\gamma^{\prime}-a}$ & $\frac{\left(\sigma_{0}^{\prime}+\gamma \mathrm{h}\right)}{\gamma^{\prime}}$ & $\frac{\left(\gamma^{\prime}-a\right)}{\gamma^{\prime}}$ \\
\hline & & $S_{B}^{\prime}$ & $\frac{\left(\sigma_{0}^{\prime}+\gamma h\right)}{\gamma^{\prime}}$ & $\frac{\left(\sigma_{0}^{\prime}+\gamma^{\prime} h\right)}{\gamma^{\prime}}$ & 1 \\
\hline C & $\mathrm{H}-\mathrm{h}_{\mathrm{c}}$ & $\mathrm{s}_{\mathrm{C}}^{\prime}$ & $\frac{\left(\sigma_{0}^{\prime}+\gamma h_{c}\right)}{\gamma^{\prime}}$ & $\frac{\left(\sigma_{0}^{\prime}+\gamma^{\prime} h_{C}\right)}{\gamma^{\prime}}$ & 1 \\
\hline
\end{tabular}

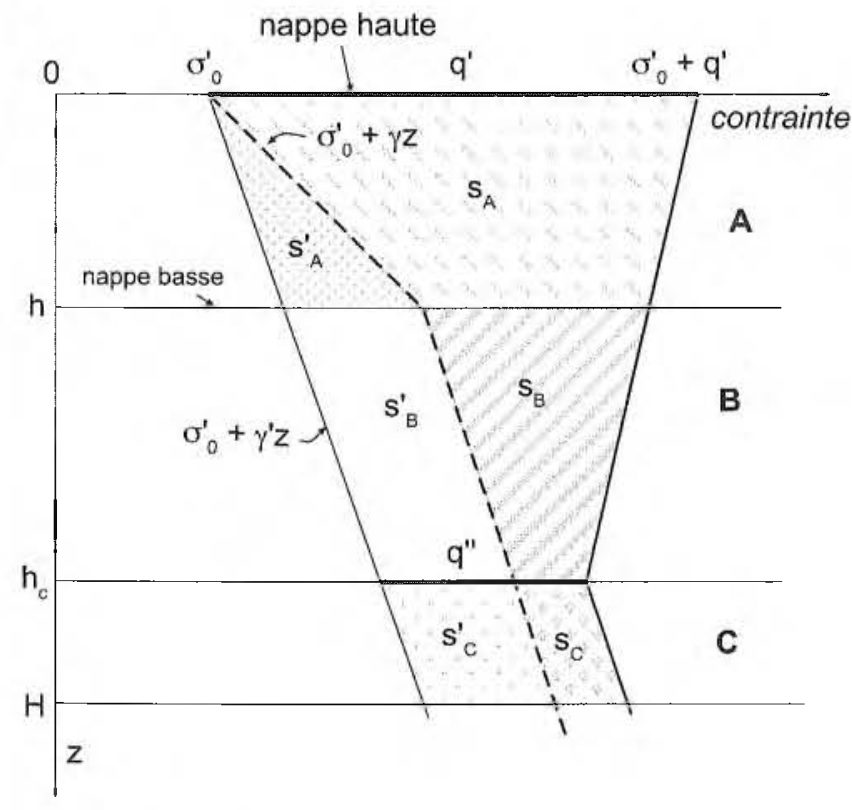

FIG. 15 Sol saturé chargé, surchargé par q(z), et nappe variable, $q^{\prime \prime}>\gamma_{w} h$ et $h<h_{c} \leq H$. Saturated loaded soil, with periodic dewatering and surcharge $\mathrm{q}(\mathrm{z})$.

\section{Exemple d'application}

On considère un sol compressible d'épaisseur $\mathrm{H}=5 \mathrm{~m}$, de caractéristiques suivantes : $\mathrm{C}_{\mathrm{c}} /\left(1+\mathrm{e}_{0}\right)=$ 0,$16 ; \mathrm{C}_{\mathrm{s}} /\left(1+\mathrm{e}_{0}\right)=0,02 ; \gamma^{\prime}=8 \mathrm{kN} / \mathrm{m}^{3}$. La nappe est en surface et baisse en période d'étiage de $\mathrm{h}=1 \mathrm{~m}$. Deux projets conduisent à prévoir des remblais d'épaisseur $\mathrm{h}=1,5 \mathrm{~m}$ et $5 \mathrm{~m}$ respectivement, de poids volumique $20 \mathrm{kN} / \mathrm{m}^{3}$, avec des impératifs de tassement très faibles.

Si on ne tient pas compte de la variation de la nappe, et si on applique la formule approchée $\mathrm{s}_{\mathrm{ap}}=\mathrm{kH}$ $\ln (1+2 \mathrm{u})$, avec $\mathrm{k}=0,07 ; \mathrm{u}=\mathrm{q} / \gamma^{\prime} \mathrm{H}=30 / 40$ et $100 / 40$ respectivement, on obtient les tassements apparents de $32 \mathrm{~cm}$ pour $\mathrm{h}_{\mathrm{r}}=1,5 \mathrm{~m}$ et $63 \mathrm{~cm}$ pour $\mathrm{h}_{\mathrm{s}}=5 \mathrm{~m}$.

En appliquant l'expression exacte (6), les valeurs de tassement deviennent respectivement $42 \mathrm{~cm}$ et $73 \mathrm{~cm}$.

Si on tient compte de la baisse de la nappe, $\mathrm{h}=1 \mathrm{~m}$, on se situe dans le cas de la figure 6 , avec pour la valeur q la plus faible, à savoir $1,5 \times 20=30 \mathrm{kPa}$, une valeur $\mathrm{h}^{\prime}$ $=30 / \gamma_{\mathrm{w}}=3 \mathrm{~m}$ supérieure à h.

On applique les expressions (18) et (19) simplifiées, puisque $\left(\sigma_{0}^{\prime}=0\right)$ et portant sur la valeur $h$, soit :

$$
\begin{gathered}
\frac{\mathrm{s}_{\mathrm{A}}}{\mathrm{kh}}=\mathrm{L}\left(\mathrm{u}_{1}, 0, \frac{\gamma^{\prime}}{\gamma}\right) \text { avec } \mathrm{u}_{1}=\frac{\mathrm{q}}{\gamma^{\prime} \mathrm{h}} \\
\frac{\mathrm{s}_{\mathrm{A}}^{\prime}}{\mathrm{k}^{\prime} \mathrm{h}}=\ln \frac{\gamma}{\gamma^{\prime}}=\mathbf{L}\left(0,0, \frac{\gamma}{\gamma^{\prime}}\right)
\end{gathered}
$$

donnant les tassements de 12 et $20 \mathrm{~cm}$ respectivement pour la couche h, soumise à la fluctuation de la nappe, dont un tassement de recompression très faible, inférieur au $\mathrm{cm}$.

Il faut ajouter à ces valeurs, les tassements des quatre mètres inférieurs, pour lesquels on applique les expressions adaptées (11) et (12) avec $\sigma_{0}^{\prime}=\gamma^{\prime} h$, puisque $q>\gamma_{w} h$

$$
u=\frac{q+\gamma^{\prime} h}{\gamma^{\prime}(H-h)}, \quad v=\frac{\gamma^{\prime} h}{\gamma^{\prime}(H-h)} \text { et } v^{\prime}=\frac{\gamma h}{\gamma^{\prime}(H-h)} \text {, }
$$

soit $\mathrm{u}=1,187$ puis $3,375, \mathrm{v}=0,25$ et $\mathrm{V}=0,563$.

On obtient pour ces quatre mètres des tassements de $15 \mathrm{~cm}$ et $39 \mathrm{~cm}$, dont 1,5 cm de recompression s'.

D'où le tassement total des deux couches : $27 \mathrm{~cm}$ pour $\mathrm{h}_{\mathrm{r}}=1,5 \mathrm{~m}$ et $59 \mathrm{~cm}$ pour $\mathrm{h}_{\mathrm{r}}=5 \mathrm{~m}$.

Si pour le remblai de $5 \mathrm{~m}$, on prévoit un réseau d'inclusions rigides composé d'éléments forés de diamètre $45 \mathrm{~cm}$ et équipés d'une tête carrée de $80 \mathrm{~cm}$ de côté 
l'espacement étant de $1,50 \mathrm{~m}$, le calcul de la contrainte résiduelle sur le sol compressible, mené suivant la méthode de Combarieu (2008), conduit à $\mathrm{q}^{\prime}=11,5 \mathrm{kPa}$ soit $11,5 \%$ de la contrainte apportée par le remblai. Cette contrainte résiduelle engendre un frottement négatif le long des inclusions, qui atteint sa valeur maximale à la profondeur $h_{c}$ de $3,5 \mathrm{~m}$ (point neutre), profondeur où, au bord de l'inclusion, la surcontrainte verticale induite est nulle. La valeur moyenne calculée de la surcontrainte verticale induite q" à cette profondeur est de $3,5 \mathrm{kPa}$ sur toute la surface d'influence de l'inclusion.

Dans ces conditions, le diagramme des contraintes montre que l'on se situe dans le cas de la figure 12 , avec $h^{\prime}=h=1 \mathrm{~m}<h_{c}=3,5<H=5 \mathrm{~m}$.

$$
\text { Le paramètre } a=\frac{q^{\prime}-q^{\prime \prime}}{h_{c}} \text { vaut } 2,29 \text {. }
$$

On peut donc appliquer les expressions du tableau III qui conduisent à $\mathrm{s}_{\mathrm{A}}=5,3 \mathrm{~cm}$.

On vérifie que les termes complémentaires de type s' sont extrêmement faibles, le tassement global atteignant $6 \mathrm{~cm}$ contre $59 \mathrm{~cm}$ sans le traitement par inclusions.
Si l'on ne tenait pas compte de la baisse de la nappe, le tassement calculé serait très largement supérieur, puisque le calcul conduit à une valeur de $16 \mathrm{~cm}$. La connaissance des variations de niveau de nappe est donc importante, car elle a une influence directe sur le dimensionnement du réseau. Les contraintes résiduelles sont en effet, au moins dans cet exemple, d'un ordre de grandeur de la contrainte de surconsolidation due à la variation de la nappe.

\section{5}

\section{Conclusion}

Pour calculer les tassements des sols compressibles sous charge de grande dimension, la méthode cedométrique est celle qu'il faut privilégier. Elle passe nécessairement par l'établissement de diagrammes, découpant le sol en couches homogènes horizontales, et comportant le tracé des contraintes, en place, passées et appliquées. Dans ces conditions, l'application des expressions fournies dans cet article permet un calcul rapide, simple, tant au niveau d'un prédimensionnement que d'un dimensionnement. L'application aux groupes d'inclusions montre la grande importance, pour le dimensionnement de ceux-ci, de la connaissance des variations du niveau de la nappe.

\section{Bibliographie}

\footnotetext{
Combarieu 0. - Amélioration des sols par inclusions rigides verticales. Application à l'édification des remblais sur sols médiocres. Revue française de géotechnique $n^{\circ} 44,1988$.

Combarieu O. - L'usage des modules de déformation en géotechnique. Revuc française de géotechnique $n^{\circ} 114,2006$.
}

Combarieu O. - Remblais sur sol compressible et inclusions rigides. Amélioration de l'approche du dimensionnement. Revue française de géotechnique $n^{\circ} 122$, 2008.
Lavisse J., Schmitt P. - Interprétation des mesures de tassement sous des remblais de préchargement au port de Rouen. Symposium intemational ASEP-GI 20u4. Presses de l'ENPC/LCPC, p. 437-445. 